\title{
Diversidad y composición de licofitas y polypodiopsidas del páramo de Anaime, Cajamarca, Tolima, Colombia
}

\author{
Lina Marcela Vargas Gaviria, Derly Astrid Buitrago, Héctor Eduardo Esquivel* \\ Grupo de Investigación en Biodiversidad y Dinámica de Ecosistemas Tropicales - GIBDET, \\ Universidad del Tolima, Ibagué, Colombia
}

\begin{abstract}
Resumen
Se hizo el estudio sobre la diversidad y la composición de licofitas y polypodiopsidas del páramo de Anaime, ubicado a $4^{\circ} 15^{\prime} \mathrm{N}$ y $75^{\circ} 33^{\prime} \mathrm{O}$, en la cordillera Central de Colombia, en un área entre los 3.200 y los 3.900 m.s.n.m., en el municipio de Cajamarca. En cinco salidas de campo se hizo el levantamiento de 16 parcelas (12 x 4,5 m). El registro de las especies y los individuos se realizó considerando las coberturas vegetales y el hábito de crecimiento. El material vegetal se procesó en el Herbario de la Universidad del Tolima (TOLI). La determinación se hizo con el apoyo de claves taxonómicas, bases de datos, la colección del TOLI y del Herbario Nacional Colombiano. En el análisis de la diversidad de especies se aplicaron los índices de riqueza de Margalef, de dominancia de Simpson y de diversidad de Shannon-Weiner. En total se registraron 50 especies distribuidas en 14 familias y 25 géneros; la familia Lycopodiaceae fue la mejor representada con 14 especies. La parcela P16 registró el mayor valor de riqueza $(\mathrm{Mg}=3,42)$; según el índice de Shannon-Weiner, las parcelas P16 y P1 tuvieron la mayor diversidad $\left(\mathrm{H}^{\prime}=3,42 ; \mathrm{H}^{\prime}=2\right.$, 95), y según el índice de Simpson, las parcelas P9 y P14 registraron la mayor dominancia $(\lambda=0,86)$. En el presente estudio se registró un incremento de 43 especies con respecto al trabajo realizado por Guerrero \& Vargas (2008), así como 25 nuevos registros para el Tolima. También se divulgó en la comunidad el catálogo elaborado, el cual contribuirá a la conservación de este páramo. (C) 2018. Acad. Colomb. Cienc. Ex. Fis. Nat.
\end{abstract}

Palabras clave: Páramo; Anaime; Licofitas; Polypodiopsidas; Diversidad; Conservación.

Diversity and composition of lycophytes and polypodiopsida of the páramo de Anaime, Cajamarca, Tolima, Colombia

\begin{abstract}
We conducted a study on the diversity and composition of lycophytes and polypodiopsida of the páramo of Anaime, which is located between $4^{\circ} 15^{\prime} \mathrm{N}$ and $75^{\circ} 33^{\prime} \mathrm{W}$ on the Central mountain range of the Colombian Andes. The area under study is located between 3200 - 3900 meters above sea level in the municipality of Cajamarca. We set-up 16 plots $(12 \mathrm{~m} \mathrm{x} 4.5 \mathrm{~m})$ in five field trips. We recorded species based on the vegetation cover and substrate classes, while the vegetal material was processed in the Universidad of Tolima Herbarium (TOLI). Species were determined using botanical keys, and the databases of TOLI and the National University Herbarium (COL). For the analysis of species diversity, we applied the Margalef index of richness, for the dominance, the Simpson index and for the diversity, the Shannon-Weiner index. We registered 50 species belonging to 14 families and 25 genus; the Lycopodiaceae family was the most representative with 14 species, and plot P16 had the highest value of species richness $(\mathrm{Mg}=3.42)$. According to the Shannon-Weiner index plots P16 and P1 had the highest diversity values $\left(H^{\prime}=3,42 ; H^{\prime}=2,95\right)$, and according to Simpson's index plots P9 and P14 had the greatest dominance $(\lambda=0.86)$. We registered an increase of 43 species compared to those registered by Guerrero \& Vargas (2008), and 25 new records for Tolima. The communication of this study in the community also included the catalog we developed, which will contribute to the conservation of the páramo. (C) 2018. Acad. Colomb. Cienc. Ex. Fis. Nat.
\end{abstract}

Key words: Páramo; Anaime; Lycophytes; Polypodiopsida; Diversity; Conservation.

\section{Introducción}

En las partes más altas de las montañas andinas, entre el límite superior de los bosques y el límite inferior de las zonas nivales, se encuentran los páramos, ecosistemas complejos y variados, endémicos de los Andes tropicales venezolanos, colombianos, ecuatorianos y peruanos (Ministerio del Medio Ambiente, 2002). Los páramos neotropicales se ubican a una altitud que va de los 3.000 a los 5.000 m.s.n.m., y se caracterizan por una temperatura media anual entre los 4 y los $10^{\circ} \mathrm{C}$, así como por notables variaciones en la

\footnotetext{
*Correspondencia:

Héctor Eduardo Esquivel, hesquive@ut.edu.co

Recibido: 16 de diciembre de 2017

Aceptado: 7 de marzo de 2018

Editor: Elizabeth Castañeda
} 
temperatura diaria; presentan suelos ricos en materia orgánica, con alta capacidad de agua, $\mathrm{pH}$ ácido y bajo contenido de fósforo disponible (Guerrero \& Vargas, 2003). En los ecosistemas de páramo es frecuente la presencia de licofitas y polypodiopsidas (equisetos y helechos), lo cual amerita el interés por conocer su composición y diversidad dada su importancia para los análisis de filogenia. Los páramos se han visto afectados por diversas actividades antrópicas como el pastoreo, la transformación del hábitat por los cultivos y de las condiciones ambientales debido al cambio climático provocado por el hombre (Van der Hammen, 1998), lo cual ha provocado la pérdida del hábitat de algunas especies de plantas $\mathbf{y}$ animales, $\mathbf{y}$ la desecación de pantanos y turberas (Rangel, 2000). En épocas de crisis como la actual, en la cual la biodiversidad está seriamente amenazada en Colombia, es esencial hacer inventarios que permitan su conocimiento, cuantificación y análisis, para así generar un programa de conservación y uso sostenible de los recursos.

Los análisis moleculares han demostrado que las pteridofitas son un grupo parafilético compuesto por dos grupos monofiléticos distintos, las licofitas (caracterizadas por poseer microfilos) y las monilofitas (actualmente llamadas polypodiopsidas, que incluyen las pteridofitas con megafilos $\mathrm{y}$ las que los perdieron). Los dos taxones son eslabones evolutivos intermedios entre las briofitas y las espermatofitas (Pryer, et al., 2001). En el análisis del ADN y de los caracteres morfológicos se ha concluido que las divisiones Psilophyta, Equisetophyta y Pterophyta constituyen un único grupo monofilético y son los parientes más cercanos de las espermatofitas.

Recientemente, un grupo de 68 investigadores de diversos centros de investigación del mundo, encabezados por Eric Schuettpelz del Instituto Smithsoniano y Harold Schneider del Museo de Historia Natural de Londres (2016), constituyeron el Grupo para la Filogenia de las Pteridofitas (Pteridophyte Phylogeny Group, PPG I), cuyo estudio, publicado en el 2016 en el Journal of Systematics and Evolution, modificó significativamente una parte de la nomenclatura que designaba algunas de las clases y subclases, y estableció el número de órdenes, familias, géneros y especies de cada grupo. En dicha publicación el grupo de investigadores presentó un cladograma de la clasificación de las licofitas y polypodiopsidas usando como primer criterio el monofilético para el reconocimiento de los taxones. Se reconocen en dicho estudio dos clases de pteridofitas: las lycopodiopsidas (licofitas) y las polypodiopsidas (helechos $\mathrm{y}$ afines), las cuales son linajes distintos dentro del árbol de las traqueofitas.

En las lycopodiopsidas se reconocen tres órdenes: las lycopodiales, las isoetales y las selagineláceas. Las primeras son de la familia Lycopodiaceae con 16 géneros y 388 especies, las segundas, de la familia Isoetaceae con un género y 250 especies, y las terceras, de la familia Sellaginelaceae con un género y 700 especies, para un total de 1.338 especies de licofitas. Las polypodiopsidas (equisetos y helechos) comprenden 11 órdenes que parten de las equisetáceas y van hasta las polypodiales, con un total de 48 familias, 319 géneros y 1.578 especies. En síntesis, el PPG I incluyó 11.916 especies distribuidas en 51 familias y 337 géneros.

En el presente estudio se hizo la determinación taxonómica de las especies de licofitas y polypodiopsidas presentes en el páramo de Anaime mediante el uso de índices de diversidad y el análisis de sus hábitos y su hábitat.

\section{Materiales y métodos}

El estudio se llevó a cabo en el páramo de Anaime (también llamado páramo de los Valles) (Figura 1), el cual se encuentra ubicado en la cordillera Central de los Andes colombianos a una latitud de $4^{\circ} 15^{\prime}$ Norte y una longitud de $75^{\circ} 30^{\prime}$ Oeste, en el rango altitudinal entre los 3.200 y los 3.900 m.s.n.m., del municipio de Cajamarca fundamentalmente, aunque también se adentra parcialmente en los municipios de Roncesvalles, Rovira e Ibagué (departamento del Tolima). En este páramo se encuentra la Reserva Natural Semillas de Agua, con algo más de 1.000 hectáreas, la cual incluye 11 quebradas, dos lagunas y extensas zonas de turberas y pantanos. Es un área natural de importancia ambiental, social y económica para más de un millón de habitantes de los departamentos del Tolima y Quindío, y se la considera una estrella hidrográfica, ya que allí nacen los ríos Anaime, Chili, Manso, Coello y Tuamo, así como fuente de abastecimiento de agua para consumo humano y de animales, para actividades de recreación y para distritos de riego. El páramo de Anaime presenta una temperatura que oscila entre $\operatorname{los} 3 \mathrm{y} \operatorname{los} 6{ }^{\circ} \mathrm{C}$, y una precipitación de 500 a $1.400 \mathrm{~mm}$ anuales. Se accede a él a través de la carretera Anaime-Santa Helena, luego de haber llegado al municipio de Cajamarca (Corporación Autónoma Regional del Tolima, 2009).

Muestreos. Se hicieron cinco salidas de campo, cada una con una duración de cuatro días. El área mínima de muestreo se estableció de acuerdo a la metodología de Rangel (1997) modificada por Marín (2013), la cual sugiere superficies de $50 \mathrm{~m}^{2}$ para los diferentes hábitats de páramo (pastizal, matorral, frailejonal y turbera). En el área de estudio se levantaron dieciséis parcelas (Figura 1 y Tabla 1), cuatro por cada hábitat, con un área de $12 \times 4.5 \mathrm{~m}$, subdividida en cuatro carriles de $3 \times 4,5 \mathrm{~m}$ para el conteo y la recolección de ejemplares. El rango de variación de las coordenadas en el área de estudio se estableció así: $4^{\circ} 16^{\prime} 0^{\prime \prime}-\mathrm{N}, 4^{\circ} 15^{\prime} 0^{\prime \prime}-\mathrm{S}$, $75^{\circ} 35^{\prime} 0$ "'-O y 75'33'0"-E, y las parcelas se ubicaron entre estos rangos.

Recolección de datos y análisis de la información. Se censaron todas las licofitas y polypodiopsidas en las dieciséis parcelas. La información de cada individuo se registró en planillas para su posterior análisis y para las determinaciones. Se calcularon los índices de diversidad alfa de Margalef, de diversidad de Shannon-Weiner y de dominancia de Simpson (Villareal, 2006). Los índices ecológicos se calcularon mediante los programas PAST (Hammer, 


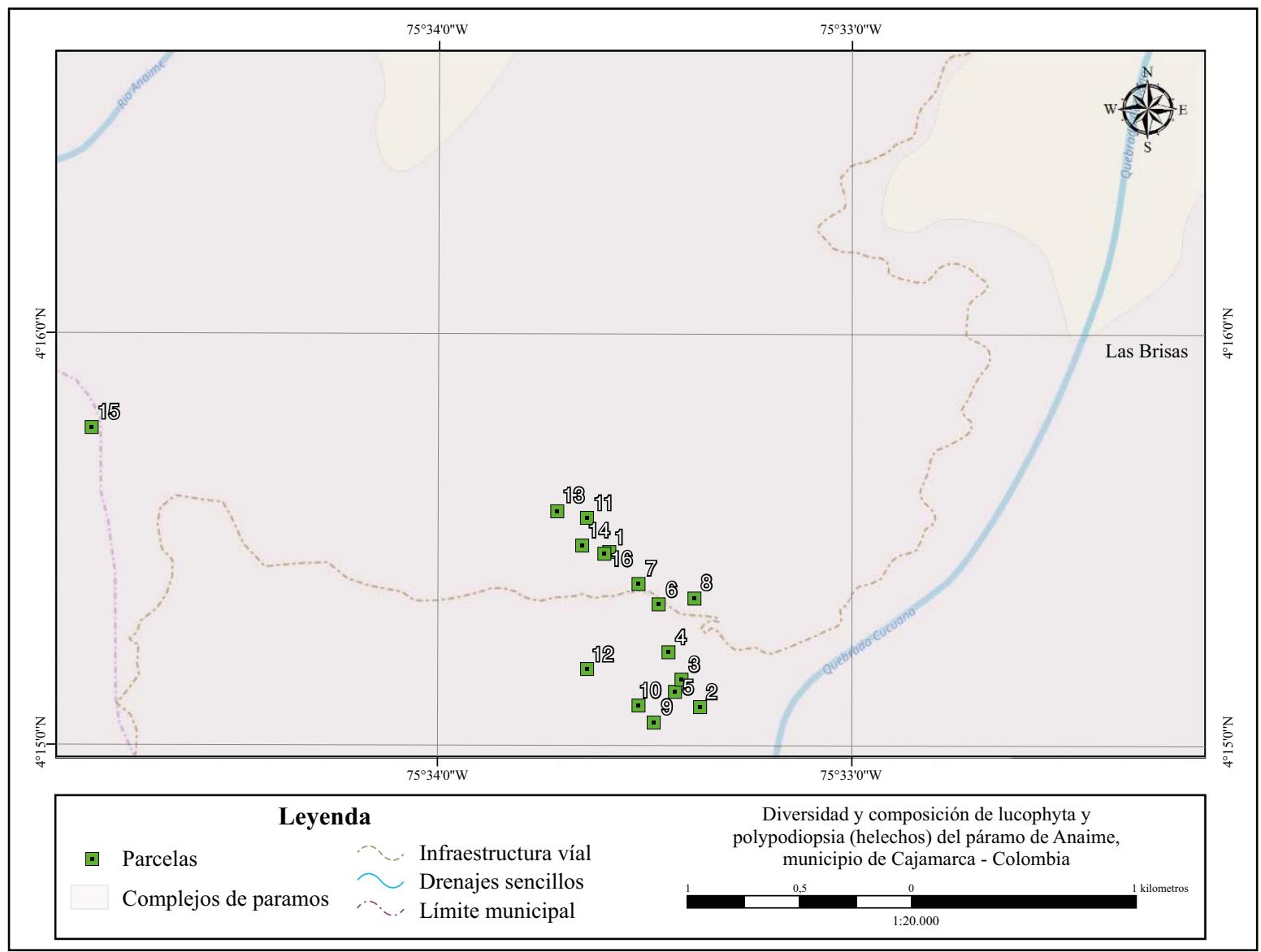

Figura 1. Mapa con la ubicación de las parcelas en el páramo de Anaime

Tabla 1. Puntos muestreados en el páramo de Anaime

\begin{tabular}{|c|c|c|c|c|c|}
\hline \multirow[t]{2}{*}{$\mathbf{N}^{\circ}$ Parcela } & \multirow{2}{*}{$\begin{array}{c}\text { Cobertura } \\
\text { vegetal }\end{array}$} & \multirow[t]{2}{*}{ Sigla } & \multirow[t]{2}{*}{ Altura (m) } & \multicolumn{2}{|c|}{ Coordenadas } \\
\hline & & & & $\mathbf{N}$ & $\mathbf{W}$ \\
\hline 1 & Frailejonal & $\mathrm{P} 1$ & 3470 & $04^{\circ} 15^{\prime} 28,2^{\prime \prime}$ & $75^{\circ} 33^{\prime} 35,1^{\prime \prime}$ \\
\hline 2 & Pastizal & $\mathrm{P} 2$ & 3570 & $04^{\circ} 15^{\prime} 5,54^{\prime \prime}$ & $75^{\circ} 33^{\prime} 21,78^{\prime \prime}$ \\
\hline 3 & Pastizal & P3 & 3543 & $04^{\circ} 15^{\prime} 9,65^{\prime \prime}$ & $75^{\circ} 33^{\prime} 24,33^{\prime \prime}$ \\
\hline 4 & Matorral & $\mathrm{P} 4$ & 3532 & $04^{\circ} 15^{\prime} 13,61^{\prime \prime}$ & $75^{\circ} 33^{\prime} 26,35^{\prime \prime}$ \\
\hline 5 & Matorral & P5 & 3557 & $04^{\circ} 15^{\prime} 7,83^{\prime \prime}$ & $75^{\circ} 33^{\prime} 25,27^{\prime \prime}$ \\
\hline 6 & Pastizal & P6 & 3579 & $04^{\circ} 15^{\prime} 20,54^{\prime \prime}$ & $75^{\circ} 33^{\prime} 27,73^{\prime \prime}$ \\
\hline 7 & Turbera & P7 & 3600 & $04^{\circ} 15^{\prime} 23,56^{\prime \prime}$ & $75^{\circ} 33^{\prime} 30,72^{\prime \prime}$ \\
\hline 8 & Frailejonal & P8 & 3600 & $04^{\circ} 15^{\prime} 21,45^{\prime \prime}$ & $75^{\circ} 33^{\prime} 22,56^{\prime \prime}$ \\
\hline 9 & Pastizal & P9 & 3600 & $04^{\circ} 15^{\prime} 3,39^{\prime \prime}$ & $75^{\circ} 33^{\prime} 28,4^{\prime \prime}$ \\
\hline 10 & Turbera & P10 & 3600 & $04^{\circ} 15^{\prime} 5,78^{\prime \prime}$ & $75^{\circ} 33^{\prime} 30,66^{\prime \prime}$ \\
\hline 11 & Turbera & P11 & 3680 & $04^{\circ} 15^{\prime} 33,14^{\prime \prime}$ & $75^{\circ} 33^{\prime} 38,2$ '” \\
\hline 12 & Matorral & P12 & 3604 & $04^{\circ} 15^{\prime} 11,16^{\prime \prime}$ & $75^{\circ} 33^{\prime} 38,1^{\prime \prime}$ \\
\hline 13 & Frailejonal & P13 & 3610 & $04^{\circ} 15^{\prime} 34,1^{\prime \prime}$ & $75^{\circ} 33^{\prime} 42,5^{\prime \prime}$ \\
\hline 14 & Frailejonal & P14 & 3582 & $04^{\circ} 15^{\prime} 29,1^{\prime \prime}$ & $75^{\circ} 33^{\prime} 38,8^{\prime \prime}$ \\
\hline 15 & Turbera & P15 & 3694 & $04^{\circ} 15^{\prime} 46,3^{\prime \prime}$ & $75^{\circ} 34^{\prime} 50,1^{\prime \prime}$ \\
\hline 16 & Matorral & P16 & 3572 & $04^{\circ} 15^{\prime} 28,04^{\prime \prime}$ & $75^{\circ} 33^{\prime} 35,6^{\prime \prime}$ \\
\hline
\end{tabular}


2001) y EstimateS 9.1 (Colwell, 2013). Los ejemplares recolectados fueron prensados y rociados con alcohol al $96 \%$ para su preservación, y posteriormente se trasladaron al Herbario de la Universidad del Tolima (TOLI).

Montaje y determinación de los ejemplares. En el TOLI se procesaron las muestras para su posterior determinación con el apoyo de claves (Murillo, M.T., Murillo, J. \& León, 2008), colecciones en línea (Herbario Virtual UNAL), bases de datos, la colección general del TOLI y el Herbario de la Universidad Nacional (COL); por último, los ejemplares se depositaron en la colección del TOLI.

Con el fin de disponer de un documento que facilitara la divulgación de los resultados en la comunidad de la cuenca del río Anaime, se elaboró un catálogo ilustrado con las especies registradas en el estudio.

\section{Resultados}

Se censaron 4.210 individuos en las 16 parcelas, con sus diferentes hábitats (pastizal, matorral, frailejonal y turbera); se registraron 50 especies pertenecientes a 25 géneros y 14 familias, 36 de las cuales correspondían a polypodiopsidas y 14 a licofitas. Las familias con el mayor número de géneros fueron la Polypodiaceae (6) y la Dryopteridaceae (4), y aquellas con el mayor número de especies fueron la Lycopodiaceae (14), la Dryopteridaceae (13), la Polypodiaceae (8) y la Pteridaceae (3) (Tabla 2).

Los ejemplares de licofitas y helechos correspondían a los siguientes hábitos: terrestre (64\%), epífita (19\%) y rupícola (17\%) (Figura 2). Elaphoglossum engelii (H.Karst.) Chris, Melpomene flabelliformis (Poir.) A.R. Sm. \& R.C. Moran y Melpomene moniliformis (Lag. ex Sw.) Proctor, se recolectaron en los tres tipos de hábito; Blechnum cordatum (Desv.) Hieron., Elaphoglossum minutum (Pohl ex Fée) T. Chris y Equisetum bogotense Kunth correspondieron a los tipos rupícola y terrestre, y Plagiogyria pectinata (Liebm.) Lellinger, al epífita y al terrestre.

Las especies que se destacaron por su mayor número de individuos fueron las siguientes: Lycopodium clavatum L. (544) (Figura 3), Plagiogyria pectinata (Liebm.)Lellinger (481), Campyloneurum angustifolium (Sw.) Fée (435), Histiopteris incisa (Thunb.) J. Sm. (394), Diphasiastrum thyoides. (Humb. \& Bonpl. ex Willd.) Holub (296), Lycopodium clavatum subsp. contiguum (Klotzsch) B. Øllg. (175) y Dryopteris wallichiana (Spreng.) Alston \& Bonner (168) (Tabla 3). Más de la mitad de las especies, se registraron únicamente en una o dos de las parcelas y solo $6,25 \%$ de ellas se hallaron en 15 de las 16 parcelas establecidas. La especie de mayor frecuencia relativa fue Lycopodium clavatum, seguida por Histiopteris incisa, Lycopodium clavatum subsp. contiguum, Hymenophyllum myriocarpum Hook y Elaphoglossum lingua (C.Presl) Brack. Por su bajo nivel de abundancia se destacaron las especies Jamesonia alstonii A.F.Tryon (Figura 4) y Blechnum loxense (Kunth) Hieron (Figura 5); entre las del género Huperzia la especie con menor abundancia fue Huperzia cruenta (Spring) Rothm (Figura 6) (Tabla 3).
Tabla 2. Familias con mayor número de especies y géneros en el páramo de Anaime

\begin{tabular}{|c|c|c|}
\hline Familia & $N^{\circ}$ de especies & $N^{\circ}$ de géneros \\
\hline Dryopteridaceae & 13 & 4 \\
\hline Polypodiaceae & 8 & 6 \\
\hline Lycopodiaceae & 14 & 3 \\
\hline Pteridaceae & 3 & 2 \\
\hline Asplenidaceae & 2 & 1 \\
\hline Blechnaceae & 2 & 1 \\
\hline Dennstaediaceae & 2 & 1 \\
\hline Athyriaceae & 1 & 1 \\
\hline Cyatheaceae & 1 & 1 \\
\hline Equisetaceae & 1 & 1 \\
\hline Hymenophyllaceae & 1 & 1 \\
\hline Lindsaeaceae & 1 & 1 \\
\hline Plagiogyriaceae & 1 & 1 \\
\hline Thelypteridaceae & 1 & 1 \\
\hline
\end{tabular}

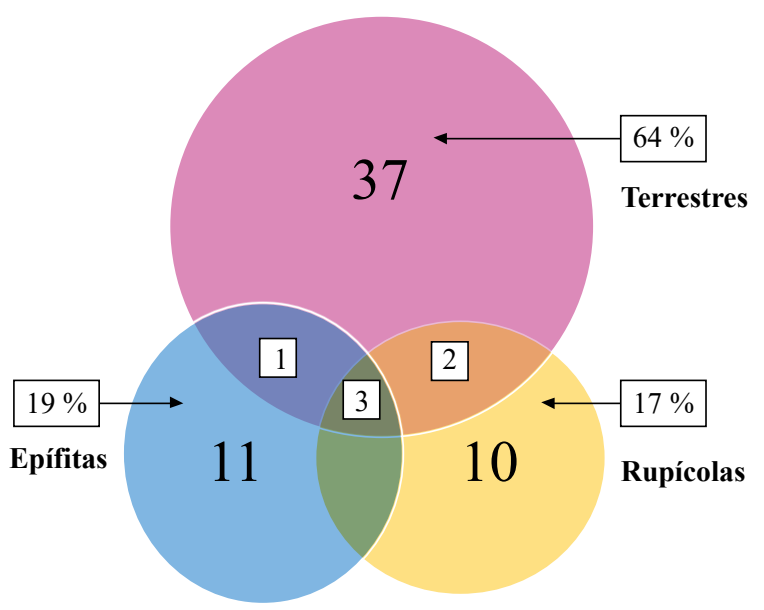

Figura 2. Distribución de las especies según el hábito de crecimiento

Según los valores del índice de Margalef, se consideró que el área estudiada presenta una riqueza media de especies de licofitas y polypodiopsidas. La parcela P16 registró el mayor valor de riqueza de especies $(\mathrm{Mg}=3,42)$, seguida por la parcela P14 (Mg=3,02), en tanto que los menores valores se encontraron en las parcelas $\mathrm{P} 10$ y $\mathrm{P} 6$, con $0,50 \mathrm{y}$ 1,39 , respectivamente. Los valores de diversidad más altos según el índice de Shannon-Weiner correspondieron a las parcelas P14 y P16 $\left(\mathrm{H}^{\prime}=2,34\right.$ y $\mathrm{H}^{\prime}=2,30$, respectivamente), seguidas de las parcelas P9 y P1 $\left(\mathrm{H}^{\prime}=2,24\right.$ y $\mathrm{H}^{\prime}=2,17$, respectivamente), en tanto que el más bajo se registró en la parcela P10 $\left(H^{\prime}=1,09\right)$. Según los valores obtenidos en el índice de Simpson, las parcelas P9 y P14 presentaron mayor dominancia $(\lambda=0,86)$, seguidas de las parcelas P2 y P5 $(\lambda=0,85)$; la menor dominancia correspondió a la P6 $(\lambda=0,53)($ Tabla 4$)$. 
Otro producto de este trabajo de investigación es el "Catálogo Ilustrado de Lycophytas y Polypodiopsidas" del páramo de Anaime, en el cual se incluyeron las 50 especies descritas brevemente, su estado de conservación y su distribución.

\section{Discusión}

El número de especies registrado para el páramo de Anaime constituye una muestra representativa de la flora de helechos y licofitas de la región paramuna en el departamento del Tolima. Los registros específicos consignados en los estudios realizados en el páramo del Ruiz (Salamanca, 2003), en la cuenca del río Combeima, en el sector del páramo del Nevado del Tolima (Esquivel \& Nieto, 2003) y en el páramo de Anaime (Guerrero \& Vargas, 2003), fueron menores a los de este, independientemente de los diferentes métodos de muestreo.

En la lista de licofitas y helechos documentada por Guerrero \& Vargas (2003) para el páramo de Anaime se registraron ocho familias, diez géneros y 17 especies, de las cuales seis especies coincidieron con el presente estudio: Blechnum loxense, Equisetum bogotense, Elaphoglossum lingua, Huperzia tetragona (Hook. \& Grev.) Trevis., Lycopodium clavatum y Jamesonia alstonii. Además, se encontró que la riqueza de taxones fue considerablemente mayor a la registrada por la Reserva Natural Semillas de Agua; 44 de las especies identificadas representan nuevos registros para el páramo de Anaime y 25 son nuevos registros para el departamento del Tolima. Cabe resaltar que son especies cuyo nicho es de alta montaña. En el estudio de Guerrero \& Vargas (2003) se citan dos especies de isoetes (Isoetes killipii Morton e Isoetes palmeri H.P. Fuchs) que no fue posible registrar en el presente estudio, a pesar de la búsqueda exhaustiva que se hizo a lo largo de las zonas de turbera.

Al confrontar estos resultados con los del estudio de Esquivel \& Nieto (2003), adelantado en un área de la misma cordillera, se observó que las especies Huperzia tetragona,

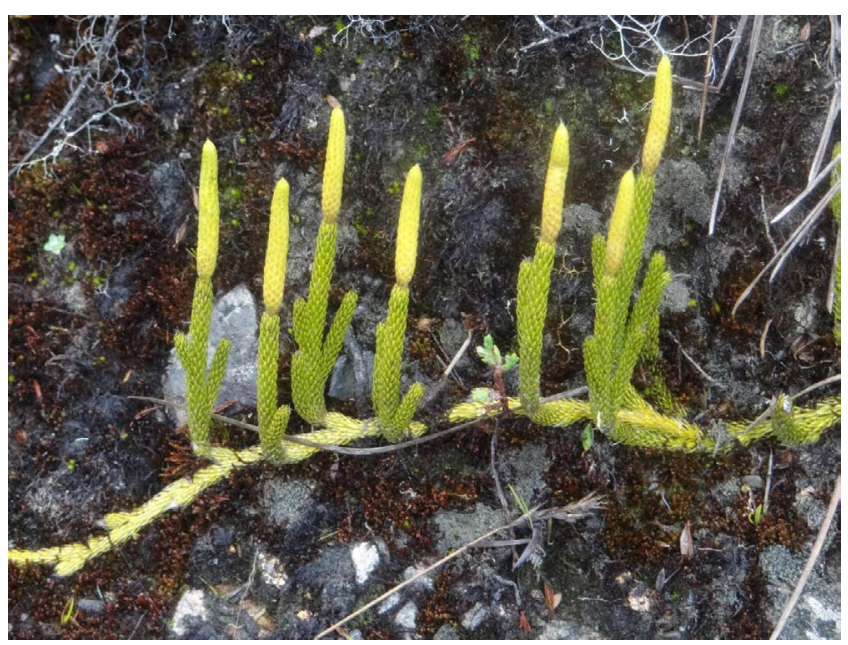

Figura 3. Lycopodium clavatum L.
Huperzia brevifolia (Grev. \& Hook.) Holub, Lycopodium clavatum, Blechnum loxense, Elaphoglossum lindenii (Bory ex Fée) Christ, Adiantum raddianum C. Presl. y Jamesonia imbricata (Sw.) Hook. \& Grev., se registraron en los dos estudios. Hay coincidencia en el registro de nueve familias, 16 géneros y 20 especies entre el estudio en el páramo de Anaime y el estudio de la cuenca del río Chinchiná-Caldas (Sanín, et al., 2008), ubicada entre los 1.000 y los 5.000 $\mathrm{m}$ de altitud sobre la vertiente occidental de la cordillera Central, en el cual se registraron 155 especies, 59 géneros y 20 familias. Esta notable diferencia en especies, géneros y familias probablemente obedece a dos razones: la primera, que el rango de estudio en la cuenca del río Chinchiná fue mucho más amplio, en tanto que en Anaime solo se evaluó el páramo, y la segunda, que la vertiente occidental de la cordillera Central tiene una mayor precipitación, ya que el régimen pluviométrico óptimo oscila entre 3.000 y 3.500 $\mathrm{mm}$, y en la vertiente oriental es de 2.000 a $2.500 \mathrm{~mm}$ como consecuencia de los flujos provenientes del Pacífico ( Van der Hammer, Pérez. \& Pinto, 1983).

El género Elaphoglossum presentó la mayor diversidad de especies (9) en el páramo de Anaime, mientras que en el Inventario de Pteridophytas de Costa Rica, Kluge \& Kessler (2005) registraron a lo largo del transecto altitudinal en el Parque Nacional Braulio Carrillo, en la Estación Biológica La Selva y en el Cerro de la Muerte, 97 especies en el rango altitudinal de los 3.100 a los $3.400 \mathrm{~m}$, siendo Elaphoglossum el género con mayor diversidad de especies. En el estudio de Carvajal \& Krömer (2015) en el centro de Veracruz (México), se registran 33 especies de la familia Polypodiaceae, en un rango de 20 a $3.500 \mathrm{~m}$, en tanto que en el presente estudio esta familia registró ocho especies correspondientes a seis géneros. Los autores del estudio en México destacan que entre los 1.500 y los $2.500 \mathrm{~m}$ los bosques son maduros y contienen la mayor diversidad de especies, pero por su ubicación en el páramo, en este trabajo la diversidad fue menor, aunque los dos estudios coinciden en la mayor diversidad de especies por familia y por género. Cabrera \& Ramírez (2014) justifican una mayor diversidad de licofitas y helechos en la cordillera Central por su alto contenido de materia orgánica y por tener un mejor estado de conservación que la cordillera Oriental, ya que en esta se presentan algunas zonas con varios relictos rodeadas por pastos limpios que alteran la continuidad y conservación de los páramos.

Rodas, Jiménez \& López (2005) analizaron en zonas boscosas del Corredor Ecológico Metropolitano, ubicado en tierras altas volcánicas del valle de Guatemala, la composición de helechos y licofitas de tres remanentes boscosos mediante la recolección de 65 especímenes de 21 especies, que no coinciden con ninguna de las especies encontradas en el páramo de Anaime.

Caviedes (1999) señala que los valores del índice de Shannon-Weiner cercanos a tres (3) o más describen comunidades muy heterogéneas; en este estudio los valores 
Tabla 3. Abundancia y frecuencia de las 50 especies registradas en el páramo de Anaime

\begin{tabular}{|c|c|c|c|c|}
\hline Especies & $\begin{array}{c}\text { Abundancia } \\
\text { absoluta }\end{array}$ & $\begin{array}{l}\text { Abundancia } \\
\text { relativa }\end{array}$ & $\begin{array}{c}\text { Frecuencia } \\
\text { Absoluta }\end{array}$ & $\begin{array}{c}\text { Frecuencia } \\
\text { Relativa }\end{array}$ \\
\hline Asplenium monanthes Murray & 6 & 0,14 & 2 & 0,99 \\
\hline *Asplenium sessilifolium Desv. & 26 & 0,61 & 3 & 1,48 \\
\hline Blechnum cordatum (Desv.) Hieron. & 26 & 0,61 & 3 & 1,48 \\
\hline Blechnum loxense (Kunth) Hieron & 3 & 0,07 & 1 & 0,49 \\
\hline Cyathea sp. & 65 & 1,54 & 6 & 2,97 \\
\hline Histiopteris incisa (Thunb.) J. Sm. & 394 & 9,35 & 11 & 5,44 \\
\hline *Sphenomeris spathulata (Maxon) K.U.Kramer & 6 & 0,14 & 2 & 0,99 \\
\hline *Elaphoglossum andreanum Christ & 21 & 0,49 & 2 & 0,99 \\
\hline *Elaphoglossum atrorubens Mickel & 26 & 0,61 & 3 & 1,48 \\
\hline *Elaphoglossum cuspidatum (Willd.) T.Moore & 26 & 0,61 & 3 & 1,48 \\
\hline Elaphoglossum engelii (H.Karst.) Christ & 65 & 1,54 & 2 & 0,99 \\
\hline *Elaphoglossum latifolium (Sw.) J.Sm & 13 & 0,30 & 1 & 0,49 \\
\hline *Elaphoglossum lindenii (Bory ex Fée) Christ & 52 & 1,23 & 4 & 1,98 \\
\hline Elaphoglossum lingua (C.Presl) Brack & 125 & 2,96 & 9 & 4,45 \\
\hline *Elaphoglossum minutum (Pohl ex Fée) Christ & 21 & 0,49 & 2 & 0,99 \\
\hline *Elaphoglossum piloselloides (L.) Keyserl. & 22 & 0,52 & 2 & 0,99 \\
\hline *Polystichum orbiculatum (Desv.) J.Rémy in Gay & 26 & 0,61 & 3 & 1,48 \\
\hline *Polystichum muricatum (L.) Fée & 8 & 0,19 & 1 & 0,49 \\
\hline *Ctenitis ampla (Humb. \& Bonpl. ex Willd.) Ching & 65 & 1,54 & 1 & 0,49 \\
\hline *Dryopteris wallichiana (Spreng.) Alston \& Bonner & 168 & 3,99 & 4 & 1,98 \\
\hline Equisetum bogotense Kunth & 79 & 1,87 & 7 & 3,46 \\
\hline *Hymenophyllum myriocarpum Hook & 90 & 2,13 & 10 & 4,95 \\
\hline *Huperzia attenuata (Spring) Trevis. & 64 & 1,52 & 7 & 3,46 \\
\hline Huperzia brevifolia (Grev. \& Hook.) Holub. & 51 & 1,21 & 4 & 1,98 \\
\hline Huperzia capellae (Herter) Holub. & 74 & 1,75 & 6 & 2,97 \\
\hline *Huperzia cruenta (Spring) Rothm. & 8 & 0,19 & 1 & 0,49 \\
\hline Huperzia hohenackeri (Herter) Holub & 14 & 0,33 & 3 & 1,48 \\
\hline Huperzia reflexa (Lam.) Rothm. & 106 & 2,51 & 4 & 1,98 \\
\hline *Huperzia rufescens (Hook.) Trevis. & 25 & 0,59 & 5 & 2,47 \\
\hline *Huperzia schlimii (Herter) B.Øllg. & 22 & 0,52 & 2 & 0,99 \\
\hline Huperzia sp & 57 & 1,35 & 2 & 0,99 \\
\hline Huperzia tetragona (Hook. \& Grev.) Trevis. & 50 & 1,18 & 2 & 0,99 \\
\hline Lycopodium clavatum $\mathrm{L}$. & 544 & 12,92 & 15 & 7,42 \\
\hline Lycopodium clavatum subsp. contiguum (Klotzsch) B.Øllg. & 175 & 4,15 & 10 & 4,95 \\
\hline Lycopodium sp. & 27 & 0,64 & 5 & 2,47 \\
\hline Diphasiastrum thyoides. (Humb. \& Bonpl. ex Willd.) Holub & 296 & 7,03 & 6 & 2,97 \\
\hline Plagiogyria semicordata (C. Presl) Christ & 481 & 11,42 & 7 & 3,46 \\
\hline Campyloneurum angustifolium (Sw.) Fée & 435 & 10,33 & 5 & 2,47 \\
\hline *Pecluma camptophyllaria (Fée) M.G.Price & 27 & 0,64 & 5 & 2,47 \\
\hline *Melpomene flabelliformis (Poir.) A.R. Sm. \& R.C. Moran & 26 & 0,61 & 4 & 1,98 \\
\hline Melpomene moniliformis (Lag. ex Sw.) A.R. Sm. \& R.C. Moran & 35 & 0,83 & 1 & 0,49 \\
\hline *Microgramma percussa (Cav.) de la Sota & 93 & 2,20 & 2 & 0,99 \\
\hline Polypodium monosorum C.Presl & 80 & 1,90 & 2 & 0,99 \\
\hline *Terpsichore lanigera (Desv.) A. R. Sm. & 36 & 0,85 & 8 & 3,96 \\
\hline *Terpsichore leucosticta (J. Sm.) A.R. Sm. & 60 & 1,42 & 1 & 0,49 \\
\hline Adiantum raddianum C. Presl. & 29 & 0,68 & 1 & 0,49 \\
\hline *Jamesonia alstonii A.F.Tryon & 1 & 0,02 & 1 & 0,49 \\
\hline Jamesonia imbricata (Sw.) Hook. \& Grev. & 14 & 0,33 & 2 & 0,99 \\
\hline *Thelypteris rudis (Kunze) Proctor & 31 & 0,73 & 3 & 1,48 \\
\hline Athyrium dombeyi Desv. & 16 & 0,38 & 6 & 2,97 \\
\hline Total & 4.210 & 100 & 202 & 100 \\
\hline
\end{tabular}

*Nuevos registros de especies para el Tolima 


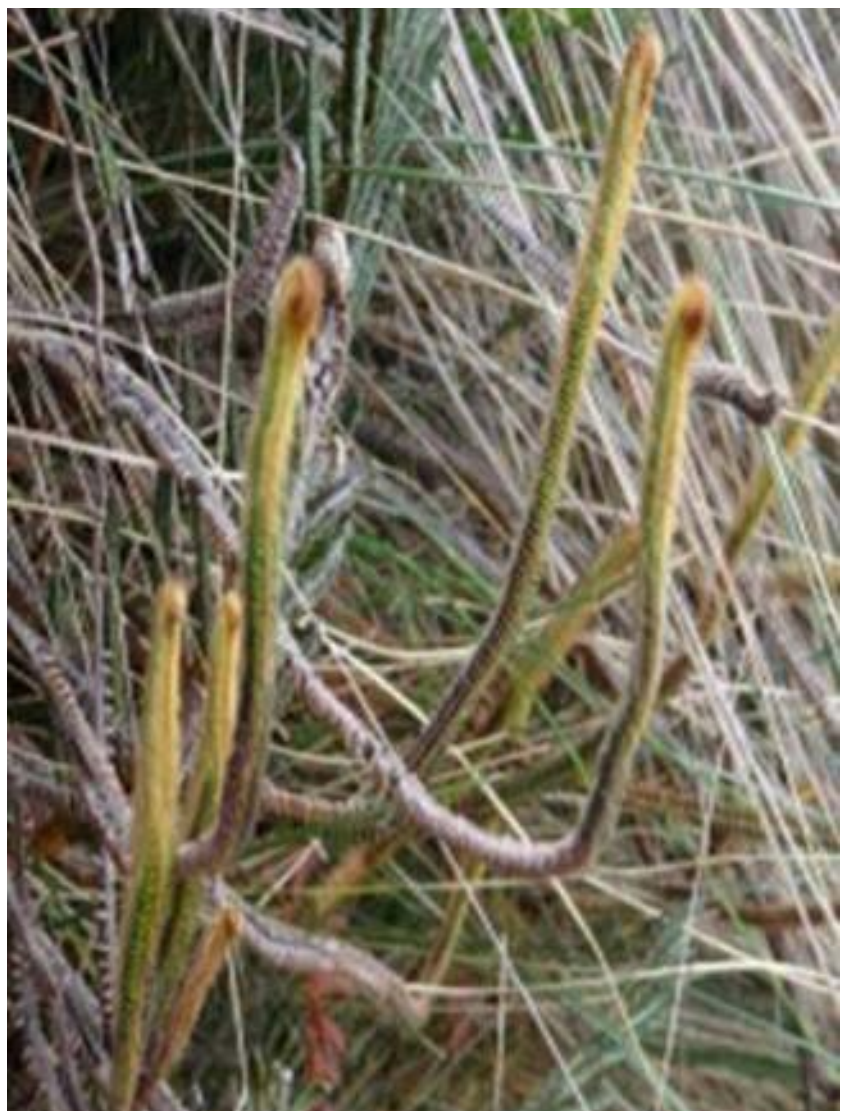

Figura 4. Jamesonia alstonii A.F.Tryon

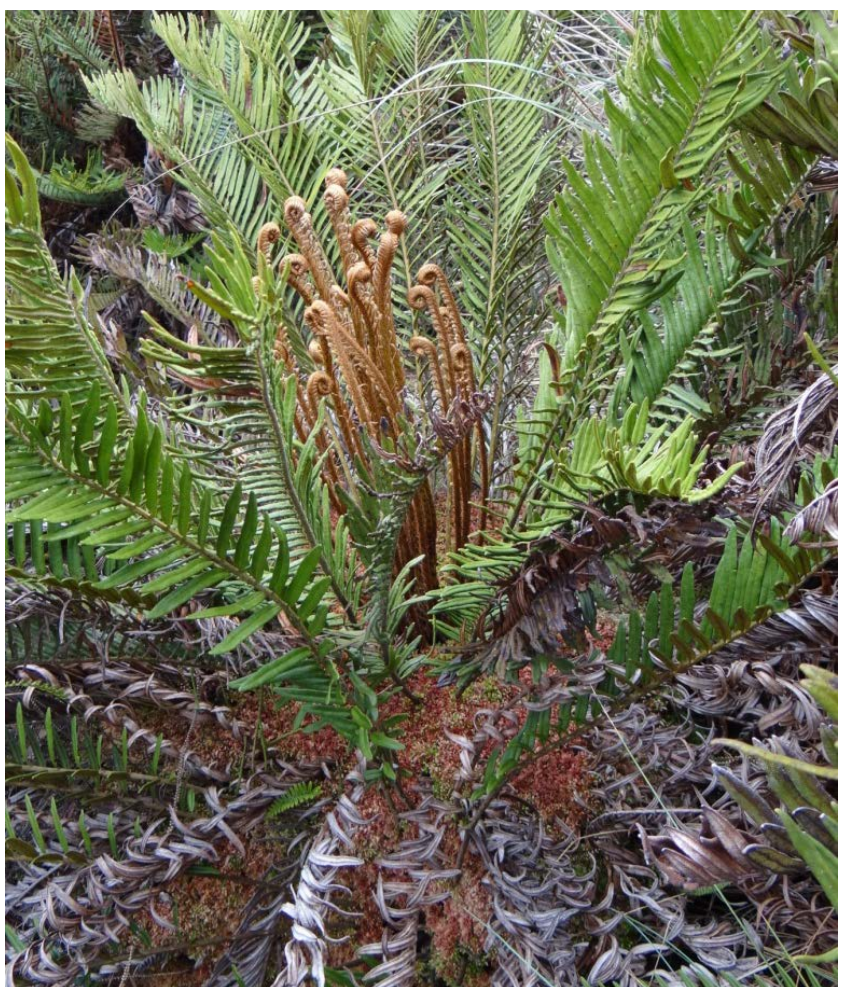

Figura 5. Blechnum loxense (Kunth) Hieron estuvieron por debajo de tres (3), lo cual evidencia que las zonas muestreadas tienen tendencia a la homogeneidad. Las parcelas P14 y P16 mostraron la mayor tendencia a la heterogeneidad, por tener valores más altos (2,34 y 2,30, respectivamente), en tanto que la mayor homogeneidad se presentó en la parcela P10, correspondiente a una turbera. Asimismo, se presentan especies dominantes que reducen la diversidad, como se evidenció en el índice de Simpson, en el cual todos los valores fueron menores de 0,9 , registrándose el mayor valor en la parcela P9 $(0,86)$, correspondiente a un hábitat de pastizal, lo cual indica que los sitios muestreados albergan una baja diversidad de especies; sin embargo, para este tipo de zonas los valores arrojados por el índice de Margalef corresponden a una riqueza media de especies, probablemente porque estas áreas están dentro del $9 \%$ de las coberturas originales que han sido intervenidas (Cabrera \& Ramírez, 2014).

Los índices para las especies de licofitas y polypodiopsidas del páramo de Anaime presentan ciertas particularidades: la parcela P16 fue la de mayor riqueza y diversidad de especies según el índice de Margalef y Shannon-Weiner, probablemente debido a la influencia de la presencia de arbustos, especialmente de las familias Asteraceae (Diplostephium sp., Ageratina tinifolia (Kunth) R.M.King \& H.Rob., Baccharis rupicola Kunth y Pentacalia vaccinioides (Kunth) Cuatrec.), Melastomataceae (Brachyotum ledifolium (Desr.) Triana, Miconia chlorocarpa Cogn y Miconia salicifolia (Bonpl. ex Naudin) Naudin) y Ericaceae (Gaultheria myrsinoides Kunth, Gaultheria sclerophylla Cuatrec. y Plutarchia monantha A.C. Sm). Las especies arbustivas antes citadas para esas tres familias hacen parte de una cobertura de matorral, lo cual pudo haber favorecido el desarrollo de diversas licofitas y polypodiopsidas, y marcaron la diferencia con respecto a las demás parcelas $(\mathrm{Mg}=3,42)$; a la parcela P16 siguió la parcela 14, correspondiente a un frailejonal $(\mathrm{Mg}=3,02)$, donde además de Espeletia hartwegiana Sch.

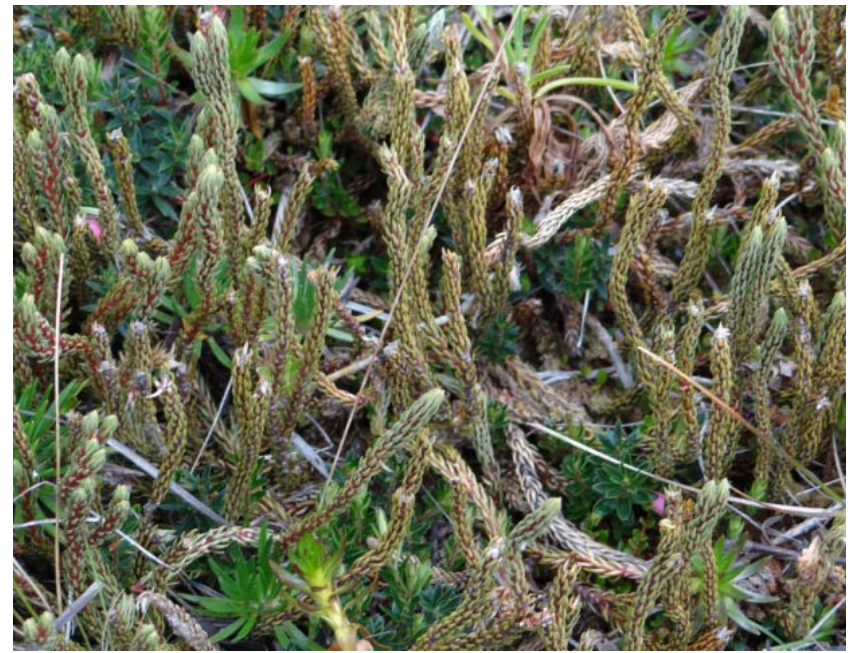

Figura 6. Huperzia cruenta (Spring) Rothm 
Tabla 4. Índices de diversidad alfa

\begin{tabular}{cccc}
\hline $\begin{array}{c}\text { Número de } \\
\text { parcelas }\end{array}$ & $\begin{array}{c}\text { Índice de } \\
\text { Margalef }\end{array}$ & $\begin{array}{c}\text { Índice de } \\
\text { Simpson }\end{array}$ & $\begin{array}{c}\text { Índice de Shannon } \\
- \text { Wiener }\end{array}$ \\
\hline 1 & 2,95 & 0,83 & 2,17 \\
\hline 2 & 2,31 & 0,85 & 2,14 \\
\hline 3 & 1,96 & 0,71 & 1,67 \\
\hline 4 & 2,63 & 0,83 & 2,15 \\
\hline 5 & 1,59 & 0,85 & 2,06 \\
\hline 6 & 1,39 & 0,53 & 1,22 \\
\hline 7 & 1,44 & 0,76 & 1,51 \\
\hline 8 & 2,59 & 0,75 & 1,98 \\
\hline 9 & 2,26 & 0,86 & 2,24 \\
\hline 10 & 0,50 & 0,66 & 1,09 \\
\hline 11 & 2,17 & 0,73 & 1,67 \\
\hline 12 & 1,54 & 0,83 & 1,94 \\
\hline 13 & 2,26 & 0,80 & 1,89 \\
\hline 14 & 3,02 & 0,86 & 2,34 \\
\hline 15 & 2,17 & 0,79 & 1,93 \\
\hline 16 & 3,42 & 0,84 & 2,30 \\
\hline & & & \\
\hline
\end{tabular}

Bip., se registró una gran influencia de los pastos del género Calamagrostis y diversas especies de Huperzia, licopodios y helechos con dominancia de Jamesonia imbricata, mientras que la turbera registró el menor valor de riqueza $(0,50)$ en la parcela P10, debido a que se trata de un área pantanosa desprovista de frailejones y matorral. En su investigación sobre la diversidad de hepáticas y musgos en turberas del Nevado del Tolima, Cataño, Uribe \& Campos (2014) referenciaron este hábitat dominado por hepáticas y musgos. Los valores de los índices para las parcelas P6 y P10 fueron los más bajos; posiblemente por estar ubicadas en zonas de pastizal y turbera.

Según los tipos de hábitos analizados, el terrestre fue el que predominó, seguido por el epífita y el rupícola; las especies comunes a los tres hábitos fueron Elaphoglossum engelii, Melpomene flabelliformis y Melpomene moniliformis, las cuales poseen una distribución cosmopolita, situación que puede estar influenciada por el requerimiento de cada una de las especies para su desarrollo, como la intensidad de la luz, la temperatura, la humedad, la cobertura del suelo, la disponibilidad de nutrientes, y las alteraciones naturales y antropogénicas de las diferentes especies (De La Sota, 1973).

\section{Conclusiones}

En el estudio se encontró que el páramo de Anaime presentó una diversidad taxonómica de 14 familias, 25 géneros y 50 especies, datos que constituyen un aporte al conocimiento de la diversidad de licofitas y polypodiopsidas en el departamento del Tolima y en Colombia, máxime si se tiene en cuenta que las cordilleras Central y Occidental han sido las menos exploradas en el país en cuanto a estos grupos se refiere (Murillo, et al., 2008).

Al comparar los resultados de este estudio con lo hasta ahora publicado para el páramo de Anaime (Guerrero \& Vargas, 2003), se registró un incremento de 44 especies pertenecientes al grupo de licofitas y polypodiopsidas y de 25 nuevos registros para el Tolima. Las licofitas y las polypodiopsidas en el páramo de Anaime están representadas principalmente por especies de las familias Dryopteridaceae y Lycopodiaceae. En cuanto a la preferencia de hábito, $64 \%$ de ellas son terrestres, $19 \%$, epífitas y $18 \%$, rupícolas.

En los muestreos del presente estudio no fue posible encontrar ejemplares de Isoetes killipii e Isoetes palmeri, de la familia Isoetaceae, registradas por Guerrero \& Vargas (2003), posiblemente debido al grado de intervención de la ganadería.

\section{Recomendaciones}

Es necesaria una mayor vigilancia de las autoridades ambientales y municipales para impedir que el páramo siga siendo afectado por actividades antrópicas como la ganadería y la minería. Asimismo, debe difundirse mediante conferencias en las instituciones educativas, las juntas comunales y entre la comunidad de la región del cañón de Anaime la información de los estudios que se han realizado para sensibilizar a los pobladores sobre la necesidad de la conservación del páramo y los bosques aledaños, puesto que es la fábrica de agua de toda esta región. También se recomienda difundir la información contenida en el catálogo ilustrado de licofitas y polypodiopsidas del páramo de Anaime para hacer conciencia en la comunidad sobre la importancia de estas especies y la necesidad de conservar el páramo. Debe reconocerse que la reserva Semillas de Agua en este páramo ha contribuido a mitigar la intervención antrópica al controlar actividades como la agricultura, la ganadería y la minería.

\section{Agradecimientos}

Los autores expresan sus agradecimientos a la Oficina de Investigaciones de la Universidad del Tolima por su apoyo económico para financiar el trabajo de campo, a la Reserva Natural Semillas de Agua por la acogida y acompañamiento en el área de estudio, al TOLI por facilitar el secado, montaje y determinación del material recolectado, al botánico José Carmelo Murillo Aldana del COL por su apoyo en la determinación de algunos especímenes, y al Laboratorio de Sistema de Información Geográfica de la Universidad del Tolima por la elaboración del mapa de ubicación de las parcelas.

\section{Contribución de los autores}

Lina Marcela Vargas Gaviria participó en las jornadas de campo, colaboró en la determinación del material vegetal, en la redacción del "Catálogo Ilustrado de Lycophytas y Monilophytas del Páramo de Anaime” y en la elaboración 
del artículo. Derly Astrid Buitrago participó en el trabajo de campo, colaboró en la determinación del material vegetal y en la redacción del Catálogo. Héctor Eduardo Esquivel participó en las jornadas de campo, coordinó todas las actividades del trabajo en campo y en el Herbario de la Universidad del Tolima y participó activamente en la elaboración del artículo.

\section{Declaración de conflicto de intereses}

Los autores declaran no tener conflicto de intereses.

\section{Referencias}

Cabrera, M., Ramírez, W. (2014). Restauración ecológica de los páramos Colombia. Transformación y herramientas para su conservación. Instituto de Investigación de Recursos Biológicos Alexander von Humboldt (IAvH). Bogotá. p. 293.

Carvajal, C., Krömer, T. (2015). Riqueza y distribución de helechos y licófitos en el gradiente altitudinal del cofre de perote, centro de Veracruz, México. Botanical Sciences. 93 (3): 601-614.

Caviedes, B. M. (1999). Manual de métodos y procedimientos estadísticos. Bogotá. Universidad Jorge Tadeo Lozano. p. 67.

Colwell, R. K. (2013). Estimate S, Version 9.1: Statistical Estimation of Species Richness and Shared Species from Samples (Software and User's Guide).

Corporación Autónoma Regional del Tolima, CORTOLIMA. (2009). Estudio de estado actual y plan de manejo de los páramos del departamento del Tolima. Tolima. Ibagué: Cortolima. Fecha de consulta: 1 de mayo de 2016. Disponible en: http://www.cortolima.gov.co/sites/default/ files/images/stories/centro_documentos/estudios/estudio del_estado_actual_de_los_paramos_en_el_epto.pdf

De La Sota, E. R. (1973). La distribución geográfica de las pteridofitas en el cono sur de América meridional. Boletín de la Sociedad Argentina de Botánica. 15 (1): 23-34.

Esquivel, H., Nieto, A. (2003). Diversidad Florística de la Cuenca Alta del Río Combeima. Ibagué: Universidad del Tolima. Ibagué. p. 190.

Guerrero, E., Vargas, W. (2003). Plantas del Páramo de Anaime. Cordillera Central, Andes Colombianos. Corporación Semillas de Agua. Cajamarca. p. 129.

Hammer, O., Harper, D., Ryan, P. (2001). PAST: Paleontological Statistics Software Package Education and Data Analysis. Palaeontología Electrónica. 4 (1): 9.

Instituto Alexander von Humboldt. (2007). Atlas de los páramos de Colombia. p. 210.

Pryer, K., Schneider, H., Smith, A., Cranfill, R., Wolf, P., Hunt, J., Sipes, S. (2001). Horsetails and ferns are a monophyletic group and the closest living relatives to seed plants. Nature. 409: 618-622.

Kluge, J., Kessler, M. (2005). Inventory of pteridophytes along an elevational transect in Braulio Carrillo National Park, La Selva Biological Station and Cerro de la Muerte, Costa Rica. Universität Göttingen Albrecht-von-Haller Institute für Pflanzenwissenschaften. Brenesia. 63-64: 11-34.

Marín, C. (2013). Propuesta metodológica para caracterizar las coberturas vegetales en los páramos de Santurbán y Rabanal. Instituto von Humboldt. Bogotá: Proyecto Páramos y Sistemas de Vida. Documento interno.
Melo, O., Vargas, R. (2003). Evaluación Ecológica y Silvicultural de Ecosistemas Boscosos. Universidad del Tolima, CRQ, CARDER, CORPOCALDAS, CORTOLIMA. 235. p. 239.

Ministerio del Medio Ambiente. (2002). Programa para el Manejo Sostenible y Restauración de Ecosistemas de la Alta Montaña colombiana. Bogotá D.C. 1: 5 .

Murillo, M.T., Murillo, J., León, A. (2008). Los Pteridófitos de Colombia. Instituto de Ciencias Naturales-Facultad de Ciencias Universidad Nacional de Colombia. Biblioteca José Jerónimo Triana. 18: 533.

Ramírez, B.R., Pinto, D.J. (2007). Catálogo de helechos y plantas afines del departamento del Cauca. Popayán, Universidad del Cauca. p. 216.

Rangel, O. (2000). La región Paramuna y Franja Aledaña en Colombia. Bogotá, Unibiblos. Colombia Diversidad Biótica III. La región de vida Paramuna. 1: 23.

Rangel, J. O., Velásquez, A. (1997). Métodos de estudio de la vegetación. Bogotá, Instituto de Ciencias Naturales, Universidad Nacional de Colombia. Colombia Diversidad Biótica II: p. 59-82.

Rodas, L., Jiménez, J., López, P. (2015). Análisis de la composición de helechos (Monilophyta y Licofitas de tres remanentes boscosos del Corredor Ecológico Metropolitano (CEM). Centro de Estudios Conservacionistas, Universidad de San Carlos de Guatemala. p. 26-34.

Salamanca, S., Cleef, A. M., Rangel, J. O. (2003). The páramo vegetation of the volcanic Ruiz-Tolima massif. Berlín, Estudios en Ecosistemas Tropandinos. 5: 82.

Sanín, D., Álvarez, L.M., Mancera, J.C., Castaño, N., González, G. (2008). Monilofitos y licofitos de la cuenca del río Chinchiná, clave para géneros y catálogo de las especies. Caldas, Rev. Acad. Colomb. Cienc. 32 (124): 331-352.

Sistema de Información Biogeográfico. (2016). Sistema de Información sobre Biodiversidad de Colombia. Fecha de consulta: 20 de enero de 2017. Disponible en: http://www. sibcolombia.net/web/sib/cifras

Smith, A. R., Pryer, K. M., Schuettpelz, E., Korall, P., Schneider, H., Wolf, P. (2006). A classification for extant ferns. Taxon. 55 (3): 705-731.

Van Der Hammen, T. (1998). Plan ambiental de la cuenca alta del río Bogotá. Bogotá. CAR. p. 142.

Schuettpelz, E., Schneider, H., Smith A.R., Hovenkamp, P., Prado, J., Germinal Rouhan, G., Xin-Mao Zhou, XM. (2016). A community-derived classification for extant lycophytes and ferns. Institute of Botany, Chinese Academy of Sciences. Journal of Systematics and Evolution. 54 (6): 563-603.

Van der Hammer, T., Pérez, A., Pinto, P. (1983). La cordillera Central Colombiana transecto parque los Nevados. J. Crammer, 1: 38.

Villarreal, H., Álvarez, M., Córdoba, S., Escobar, F., Fagua, G., Gast, F., Mendoza, H., Ospina, M., Umaña, A.M. (2006). Manual de métodos para el desarrollo de inventarios de biodiversidad. Bogotá, Programa de Inventarios de Biodiversidad. Instituto de Investigación de Recursos Biológicos Alexander von Humboldt. 2: 236. 